\title{
The Effect of Sleep Disturbance on Quality of Life in Pregnant Women
}

\author{
Dilek Celikgoz and Gul Pinar* \\ Department of Nursing, Yildirim Beyazit University Faculty of Health Sciences, Ankara, Turkey
}

\begin{abstract}
Background: Most women are at risk for some degree of sleep disturbance (insomnia) during pregnancy because of the hormonal and physical changes, which may negatively affect women's quality of life.

Aim: This study was carried out to determine the sleep disorders of pregnant women and to examine the effect of these disorders on their quality of life.

Method: This cross-sectional and descriptive study conducted in a State University Hospital Obstetrics Clinic in Ankara, Turkey. In the collection of data, Individual Information Form, Pittsburgh Sleep Quality Index and SF-36 Quality of Life Scale were used. To analyze the collection data, Man Whitney $U$ test, Kruskall Wallis test and Pearson correlation test were applied.
\end{abstract}

Results: $68.5 \%$ of women experienced insomnia. Insomnia was linked to a lower quality of life $(p<0.05)$. Advanced age, low education, low income, obesity, co-morbidity, multi-parity, multi-gravida, advanced gestational age, in-sufficient antenatal care and social support, sedentary life-style, drinking of tea and cigarette habit has been associated with poor sleep quality $(p<0.05)$.

Conclusion: Women suffered from insomnia during pregnancy, which affects quality of life adversely. Therefore, assessment of sleep should be an integral part of prenatal care. Further research is required to determine quality of life effect on sleep.

\section{Keywords}

Pregnancy, Sleep quality, Sleep disorder, Insomnia, Quality of life

\section{Introduction}

\section{Definition of the problem}

Pregnancy is the most enjoyable and most sensitive period of a women's life. Even in uncomplicated pregnancies, this period is associated with hormonal, metabolic, physiological, anatomical, and psychological changes, which may negatively affect the quality of life [1-4]. Insomnia is very common in all stages of pregnancy, which is defined both as a symptom and as a disorder [5-13]. Pregnancy-related issues such as frequent urination, nausea, vomiting, anxiety, stress, heartburn, nasal congestion, back pain, fatigue, leg cramps, abdominal discomfort, and growing fetus can contribute to sleep problems and can also impair a pregnant woman's quality of life [2,9,14-21].

Insomnia in pregnancy is treatable condition with pharmacological interventions, also alternative methods or behavioral strategies may alleviate sleep disturbance. Minimizing modifiable factors for insomnia and increasing sleep quality play an essential role to avoid adverse pregnancy outcomes such as maternal-fetal morbidity, mortality, anxiety, depression, and cognitive impairments [22-26]. Despite all these potential adverse effects, few studies have focused on insomnia as a contributing factor to the deterioration of quality of life.

\section{Aim}

This study was conducted to investigate insomnia in pregnant women and its effect on quality of life.

*Corresponding author: Assoc. Prof. Gul Pinar, Department of Nursing, Yildirim Beyazit University Faculty of Health Sciences, Cankirisk, Cicek cd. No:3/2, 06610 Ulus, Ankara, Turkey, Tel: $+9(0)-312-324155 / 4556$

Accepted: June 05, 2021

Published online: June 07, 2021

Citation: Celikgoz D, Pinar G (2021) The Effect of Sleep Disturbance on Quality of Life in Pregnant Women. J Nurs Pract 4(1):293-301 


\section{Hypotheses}

$H_{1}$ : The quality of life in pregnant women with insomnia changes negatively.

$\mathrm{H}_{0}$ : There is no change in the quality of life of pregnant women with insomnia.

\section{Materials and Methods}

\section{Study type, design, setting, period, population, and sample size}

This descriptive and cross-sectional study was conducted during June-July 2015 in a State University Hospital, Obstetric Clinic in Ankara, Turkey. The universe of the study consisted of 1000 women who applied to the obstetric clinic of the hospital for routine control (in the last year). The sample of the study was determined according to the formula "calculating the sample size in case of a known universe" (n $=\mathrm{t}^{2} \cdot \mathrm{p} \cdot \mathrm{q} / \mathrm{d}^{2}$ ) the sample size was determined as 267 pregnant women $(95 \% \mathrm{Cl}$, alpha $=0.05)$ [27].

\section{Sampling selection criteria}

1) Those who volunteer to participate in the study, 2) Who do not have an auditory or mental disability, 3) Who were open to cooperation and able to understand in Turkish language, 4) Who do not have a health problem requiring hospitalization, 5) Pregnant women applying to the polyclinic for routine control, and 6) Who do not have twin or triplet pregnancies.

\section{Dependent and independent variables}

Dependent variables; sleep quality and quality of life scores.

Independent variables; 1) Socio-demographic characteristics of pregnant women (age, educational status, employment status, working status before pregnancy, income status, presence of social security, family type), 2) Medical and pregnancy-related characteristics (weight, height, weight before pregnancy, regularly used drugs, pregnancy week, existence of chronic disease, whether pregnancy is planned, number of pregnancies, number of living children, status of receiving social support, status of getting ready for childbirth education and health problems that occur with pregnancy, 3) Habits (smoking, alcohol, tea-coffee consumption, and exercising regularly).

\section{Instruments}

Three separate data collections: Individual Information Form, Pittsburgh Sleep Quality Index (PSQI) and SF-36 Quality of Life Scale (QLS) tool was applied by the researchers using face-to-face interview method. It took an average of 5-10 minutes to complete.

Individual information form: This form was prepared by the researchers based on the literature review [1-19]. The form consists of 3 parts and 32 questions.

1. Socio-demographic characteristics such as age, education, employment, income, social security and family type etc.
2. Medical and pregnancy-related characteristics such as BMI, co-morbidity, regularly used drugs, gestational age, planned pregnancy, gravida, parity.

3. Sleep characteristics and other habits such as cigarette/ alcohol habits, drinking tea and coffee, regular exercise during pregnancy, sleep problems in the past and current pregnancy, receiving health services, and cope with their sleep problems.

Pittsburgh sleep quality index-PSQI: The PSQI was developed by Buysse, et al. [28] to assess sleep quality in 1988. It was adapted to in this study, the reliability coefficient of the scale was found to be 0.86 . In scoring the PSQI, the measure consists of 19 individual items, and seven component scores are derived. Each scored 0 (no difficulty) to 3 (severe difficulty) that produce one global score ranging from 0 to 21 , where lower scores denote a healthier sleep quality. The cutoff point is five, and sleep quality is classified as good (0-4 points), sleep quality as poor (5-21 points).

The SF-36 quality of life scale-QLS: The SF-36 QLS was developed by Rand Corporation in 1992 to measure of health status. Crohnbach's alpha reliability coefficient of the scale was found to be 0.89 [29]. In this study, the reliability coefficient of the scale was found to be 0.94 . The scale has 36 question items and eight sub-dimensions; physical function (10 items), social function ( 2 items), physical role function (4 items), emotional well being ( 3 items), mental health ( 5 items), energy/vitality ( 4 items), pain ( 2 items) and general health perception ( 5 items). The scale is evaluated considering the last 4 weeks. The lowest score that can be obtained from the scale is 0 and the highest score is 100 . According to the original scale the higher the score, the higher the quality of life.

\section{Ethical approval}

Ethical approval was obtained for the conduct of this study from the Research and Ethics Committee of the hospital. (No: KO11/00). The researcher introduced the questionnaire to participants and explained the coverage of the material. Participants completed an informed consent form in which they were assured of the confidentiality of their responses following which they provided informed consent that participation was voluntary and anonymous. Rules specified in the Helsinki Declaration were observed in the data collection phase.

\section{Data analyses}

Descriptive statistics were calculated for all variables. In the evaluation of the data, besides the number, percentage, mean and standard deviation representations of the variables, the Mann-Whitney $U$ test was used when the data was not normally distributed in the two-group comparisons, the Kruskall-Wallis test was used in the comparisons with three or more groups, and the Pearson correlation test was used to examine the relationship between the two scales. A value of $\mathrm{P}<0.05$ was considered statistically significant.

\section{Results}

The average age of patients was $27.5 \pm 4.9$ years 
Table 1: Distribution of socio-demographic and habits characteristics.

\begin{tabular}{|c|c|c|}
\hline Socio-demographic-habits characteristics & n & $\%$ \\
\hline \multicolumn{3}{|c|}{ Age (Mean $=27.5 \pm 4.9$ years, $\min : 17, \max : 42)$} \\
\hline $17-24$ & 81 & 30.3 \\
\hline $25-29$ & 90 & 33.7 \\
\hline$\geq 30$ & 96 & 35.9 \\
\hline \multicolumn{3}{|l|}{ Education } \\
\hline Primary school & 114 & 42.7 \\
\hline High school & 109 & 40.8 \\
\hline University and above & 44 & 16.5 \\
\hline \multicolumn{3}{|l|}{ Employment } \\
\hline Working & 86 & 32.2 \\
\hline Housewife & 181 & 67.8 \\
\hline \multicolumn{3}{|l|}{ Income } \\
\hline Poor & 74 & 27.7 \\
\hline Moderate & 152 & 56.9 \\
\hline Good & 41 & 15.4 \\
\hline \multicolumn{3}{|l|}{ Body mass index $\left(\mathrm{kg} / \mathrm{m}^{2}\right)$ pre-pregnancy } \\
\hline Normal (17-24.9) & 90 & 33.7 \\
\hline Overweight (25-29.9) & 122 & 45.7 \\
\hline Obesity (30-34) & 55 & 20.6 \\
\hline \multicolumn{3}{|l|}{ Family type } \\
\hline Extend & 73 & 27.3 \\
\hline Nuclear & 194 & 72.7 \\
\hline \multicolumn{3}{|l|}{ Smoker (pre-pregnancy-n = 45, 16.9\%) } \\
\hline Yes (still in pregnancy) & 15 & 5.6 \\
\hline No & 252 & 94.4 \\
\hline \multicolumn{3}{|l|}{ Regular exercises } \\
\hline Yes & 78 & 29.2 \\
\hline No & 189 & 70.8 \\
\hline \multicolumn{3}{|l|}{ Regular drinking coffee } \\
\hline Yes & 40 & 15 \\
\hline No & 227 & 85 \\
\hline \multicolumn{3}{|l|}{ Regular drinking tea } \\
\hline Yes & 219 & 80 \\
\hline No & 48 & 20 \\
\hline \multicolumn{3}{|l|}{ Regular used medication } \\
\hline Yes & 53 & 19.9 \\
\hline No & 214 & 80.1 \\
\hline Total & 267 & 100.0 \\
\hline
\end{tabular}

(min: 17, Max: 42). Some socio-demographic and medical characteristics and habits of pregnant women are presented in Table $1.62 .6 \%$ of the pregnant women were in their third trimester, $37.5 \%$ had their second pregnancy, $41.2 \%$ had a living child, $73 \%$ had a planned pregnancy, $82 \%$ received support from their spouse or social environment, $26.6 \%$ had received antenatal training, and $9.7 \%$ had pregnancy-related
Table 2: Distribution of pregnancy-related characteristics.

\begin{tabular}{|c|c|c|}
\hline Pregnancy-related characteristics & $\mathbf{n}$ & $\%$ \\
\hline \multicolumn{3}{|l|}{ Gestational age } \\
\hline I. Trimester (1-13. wk) & 33 & 12.4 \\
\hline II. Trimester (14-26. wk) & 67 & 25.0 \\
\hline III. Trimester (27-41. wk) & 167 & 62.6 \\
\hline \multicolumn{3}{|l|}{ Planned pregnancy } \\
\hline Yes & 195 & 73.0 \\
\hline No & 72 & 27.0 \\
\hline \multicolumn{3}{|l|}{ Gravida } \\
\hline 1 & 97 & 36.3 \\
\hline 2 & 100 & 37.5 \\
\hline$\geq 3$ & 70 & 26.2 \\
\hline \multicolumn{3}{|l|}{ Parity } \\
\hline 0 & 100 & 37.5 \\
\hline 1 & 110 & 41.2 \\
\hline 2 & 46 & 17.2 \\
\hline$\geq 3$ & 11 & 4.1 \\
\hline \multicolumn{3}{|l|}{ Receiving social support } \\
\hline Yes & 219 & 82.0 \\
\hline No & 23 & 8.6 \\
\hline Partially & 25 & 9.4 \\
\hline \multicolumn{3}{|l|}{ Receiving antenatal education } \\
\hline Yes & 71 & 26.6 \\
\hline No & 196 & 73.4 \\
\hline \multicolumn{3}{|l|}{ Pre-pregnancy diseases } \\
\hline Yes $^{*}$ & 18 & 6.7 \\
\hline No & 249 & 93.3 \\
\hline \multicolumn{3}{|c|}{ Pregnancy-related complaints ( $n=80 \%$ ) } \\
\hline Tiredness-fatigue & 186 & 69.7 \\
\hline Frequent urination & 157 & 58.8 \\
\hline Nausea-vomiting & 153 & 57.3 \\
\hline Leg cramps & 150 & 56.2 \\
\hline Nervousness & 121 & 45.3 \\
\hline Back-pain & 107 & 40.1 \\
\hline Swelling-gas & 90 & 33.7 \\
\hline Respiratory distress & 82 & 30.7 \\
\hline Restless leg syndrome & 22 & 8.3 \\
\hline \multicolumn{3}{|l|}{ Pregnancy-related diseases } \\
\hline Yes $^{* *}$ & 26 & 9.7 \\
\hline No & 241 & 90.3 \\
\hline
\end{tabular}

*Thyroid $38.9 \%$, asthma $16.7 \%$, diabetes $16.7 \%$, hepatitis B $16.7 \%$, rheumatism $11.2 \%$; ${ }^{* *}$ Thyroid $53.8 \%$, hypertension $17.3 \%$, migraine $15.3 \%$, and diabetes $11.5 \%$.

disease during this period. Reported diseases were as follows, thyroid $(53.8 \%)$, hypertension $(17.3 \%)$, migraine (15.3\%), and diabetes (11.5\%). Women declaring pregnancy-related 
complaints more commonly suffered from fatigue $(69.7 \%)$, frequent urination (58.8\%), nausea-vomiting (57.3\%), legcramps $(56.2 \%)$, tension-nervousness $(45.3 \%)$, back-pain (40.1\%), abdominal swelling (33.7\%), respiratory distress $(30.7 \%)$ and restless leg syndrome (8.3\%), (Table 2$)$.

In Table 3, it was seen that $2.2 \%$ of pregnant women had insomnia in the past, $53.9 \%$ of them had insomnia (subjective) in their current pregnancy, and $6.2 \%$ of those who applied to health institutions for sleep problems. The most common reasons for insomnia were as follows, advanced gestational age $(20.5 \%)$, bodily pain $(15.6 \%)$, baby movements $(12.5 \%)$, stress (9.4\%), gastrological problems (reflux) (8.3\%), fever (7.3\%), and weight gain (7.8\%). Pregnant women frequently

Table 3: Distribution of sleep-related characteristics.

\begin{tabular}{|c|c|c|}
\hline Sleep-related characteristics & n & $\%$ \\
\hline \multicolumn{3}{|l|}{ Insomnia in the past } \\
\hline Yes & 6 & 2.2 \\
\hline No & 261 & 97.8 \\
\hline \multicolumn{3}{|c|}{ Insomnia during pregnancy (self-reported) } \\
\hline Yes & 144 & 53.9 \\
\hline No & 123 & 46.1 \\
\hline \multicolumn{3}{|l|}{ Reasons for insomnia $(n=144)$} \\
\hline Advanced pregnancy week & 39 & 20.5 \\
\hline Back-pain & 30 & 15.6 \\
\hline Baby movements & 24 & 12.5 \\
\hline Stress & 18 & 9.4 \\
\hline Stomach problems (reflux) & 16 & 8.3 \\
\hline Weight gain & 15 & 7.8 \\
\hline Fever & 14 & 7.3 \\
\hline Frequent urination & 13 & 6.8 \\
\hline Tiredness & 13 & 6.8 \\
\hline Respiratory distress & 6 & 3.1 \\
\hline Irritability & 4 & 2.0 \\
\hline \multicolumn{3}{|c|}{ Receiving health service for insomnia } \\
\hline Yes & 9 & 6.2 \\
\hline No & 135 & 93.8 \\
\hline \multicolumn{3}{|l|}{ Persistence of insomnia } \\
\hline Yes & 128 & 87.7 \\
\hline No & 16 & 12.3 \\
\hline \multicolumn{3}{|l|}{ Dealing with sleep problems } \\
\hline Take a warm shower & 53 & 36.8 \\
\hline Watch TV & 45 & 31.3 \\
\hline Drinking warm milk & 42 & 29.2 \\
\hline Reading books & 17 & 11.8 \\
\hline Listen to music & 14 & 9.7 \\
\hline Drinking herbal tea & 2 & 1.4 \\
\hline Increasing the number of pillows & 2 & 1.4 \\
\hline Not doing anything & 51 & 35.4 \\
\hline
\end{tabular}

used a warm shower (36.8\%), watching television (31.3\%) and drinking a warm milk to cope with insomnia (29.2\%). Some of them were doing nothing (35.4\%).

In Table 4, the mean global score of the PSQI was $6.8 \pm$ $3.6(95 \% \mathrm{Cl})$. The mean global score of the SF-36 QLS was 32 \pm 12 . The subgroups of the scale, respectively, physical function score was $36.9 \pm 14.6$, role difficulty (physical) score average was $21.6 \pm 20.5$, pain score average was $33.8 \pm 18.5$, general health score average was $35.3 \pm 9.5$, vitality (energy) score average was $34.1 \pm 15.3$, social function score average was $34.6 \pm 14.7$, role difficulty (emotional) score was $25.3 \pm 20.0$, and mental health score was $36.3 \pm 10.4$. According to the SF36 QLS, the quality of life of the pregnant women was below the average. There was a negative correlation between the scores of pregnant women from PSQI and the sub-dimension of life quality scale $(r=-0.386, p=0.000)$, (Table 4). Also, the mean scores of physical function and role difficulties were decreasing as the insomnia increased (Figure 1).

Table 5 shows the total PSQI scores of pregnant women and their subscale scores in the last month. Even though the daily sleep duration of the pregnant women (mean: 7.2 hours \pm 3.4 ) was found to be within normal limits, more than half of them had insomnia, which indicates their low quality of sleep. According to the PSQI, $68.5 \%$ of the pregnant women had insomnia. It is seen that the times of falling a sleep of most of the women with insomnia were longer than those with good sleep quality $(p<0.001)$. Women with good sleep quality have longer periods of sleep $(p<0.001)$.

In Table 6, several factors were determined to have an independent effect on sleep problems. There was a significant difference between age groups in terms of sleep quality, duration, efficiency and sleep disorder component ( $p$ $<0.05$ ). Accordingly, it was observed that the older age group had more sleep problems. Sleep latency (difficulty falling asleep) is significantly higher in low-income groups $(p<0.05)$. Sleep duration, sleep disturbance, daytime dysfunction, global score were found to be significantly higher in those with co-morbidity than those without the diseases $(p<0.05)$. A stronger relationship was observed between obesity and insomnia $(p<0.05)$. As the week of gestation increased, sleep duration decreased, sleep quality decreased $(p<0.05)$. Women with high parity had difficulty falling asleep $(p<0.05)$. Sleep quality, sleep latency, sleep duration, sleep efficiency, sleep disturbance, daytime dysfunction and global score scores of those who had pregnancy-related problems during pregnancy were found to be significantly higher than those who did not have pregnancy-related problems $(p<0.05)$. Smoking increased the risk of sleep problems (sleep latency, sleep disturbance and general score) $(p<0.05)$. Physical activity was associated with decreased sleep problems (sleep quality, sleep efficiency and global score) ( $p<0.05$ ). The sleep quality of women receiving antenatal care and social support was found to be high, but who regularly consumed the tea was found to be low $(p<0.05)$, (Table 6). However, no significant difference was found between insomnia and family type, occupation, and coffee consumption (during pregnancy is very low) $(p>0.05)$. 
Table 4: Descriptive characteristics of PSQI-SF-36 QOL scores and scales correlation.

\begin{tabular}{|c|c|c|c|c|c|c|c|c|c|c|c|}
\hline \multicolumn{4}{|c|}{ PSQI - Subscales (0-21) } & \multirow{2}{*}{\multicolumn{3}{|c|}{\begin{tabular}{|l|} 
Mean \\
1.2 \\
\end{tabular}}} & \multirow{2}{*}{\multicolumn{2}{|c|}{\begin{tabular}{|l|} 
Min \\
0.0 \\
\end{tabular}}} & \multirow{2}{*}{\begin{tabular}{|l} 
Max \\
3.0
\end{tabular}} & \multirow{2}{*}{\multicolumn{2}{|c|}{\begin{tabular}{|l|} 
SD $^{*}$ \\
0.9 \\
\end{tabular}}} \\
\hline 1. & \multicolumn{3}{|c|}{ Sleep quality } & & & & & & & & \\
\hline 2. & \multicolumn{3}{|c|}{ Sleep latency } & \multicolumn{3}{|l|}{1.4} & \multicolumn{2}{|l|}{0.0} & 3.0 & \multicolumn{2}{|l|}{0.9} \\
\hline 3. & \multicolumn{3}{|c|}{ Sleep duration } & \multicolumn{3}{|l|}{0.6} & \multicolumn{2}{|l|}{0.0} & 3.0 & \multicolumn{2}{|l|}{0.8} \\
\hline 4. & \multicolumn{3}{|c|}{ Habitual sleep efficiency } & \multicolumn{3}{|l|}{0.7} & \multicolumn{2}{|l|}{0.0} & 3.0 & \multicolumn{2}{|l|}{1.1} \\
\hline 5. & \multicolumn{3}{|c|}{ Sleep disturbance } & \multicolumn{3}{|l|}{1.6} & \multicolumn{2}{|l|}{1.0} & 3.0 & \multicolumn{2}{|l|}{0.5} \\
\hline 6. & \multicolumn{3}{|c|}{ Use of sleeping medication } & \multicolumn{3}{|l|}{0.0} & \multicolumn{2}{|l|}{0.0} & 1.0 & \multicolumn{2}{|l|}{0.1} \\
\hline 7. & \multicolumn{3}{|c|}{ Daytime dysfunction } & 0.9 & & & 0.0 & & 3.0 & 0.8 & \\
\hline Globa & ore & & & 6.8 & & & 0.0 & & 17 & 3.6 & \\
\hline SF-36 & L- Subsc & es $(0-100)$ & & Mean & & & Min & & Max & SD & \\
\hline 1. & Physica & unction & & 36.9 & & & 0.0 & & 66.7 & 14.6 & \\
\hline 2. & Role dif & ulty (physic & & 21.6 & & & 0.0 & & 62.5 & 20.5 & \\
\hline 3. & Pain & & & 33.8 & & & 0.0 & & 81.8 & 18.5 & \\
\hline 4. & Genera & ealth & & 35.3 & & & 0.0 & & 60.0 & 9.5 & \\
\hline 5. & Vitality & nergy) & & 34.1 & & & 0.0 & & 66.7 & 15.3 & \\
\hline 6. & Social $f$ & ction & & 34.6 & & & 0.0 & & 80.0 & 14.7 & \\
\hline 7. & Role dif & ulty (emotic & & 25.3 & & & 0.0 & & 50.0 & 20.0 & \\
\hline 8. & Mental & ealth & & 36.3 & & & 0.0 & & 63.3 & 10.4 & \\
\hline Globa & ore & & & 32.4 & & & 0.0 & & 74.3 & 12.8 & \\
\hline & & & & & QOL scal & & & & & & \\
\hline & & 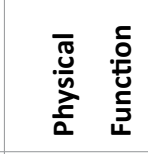 & 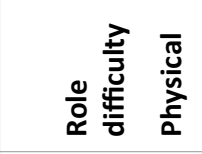 & 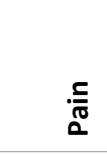 & 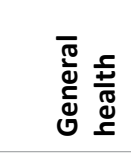 & 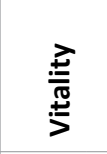 & & 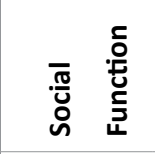 & 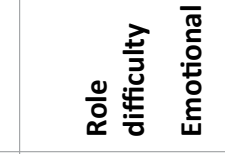 & $\begin{array}{l}\frac{\pi}{2} \\
\stackrel{0}{\Sigma}\end{array}$ & $\begin{array}{l}\frac{5}{\pi} \\
\frac{\pi}{1}\end{array}$ \\
\hline PSQI & $r$ & $-0.322^{*}$ & $-0.280^{*}$ & $0.386^{*}$ & 0.116 & $0.140^{*}$ & & -0.098 & $-0.251^{*}$ & 0.027 & \\
\hline & $p$ & 0.000 & 0.000 & 0.000 & 0.059 & 0.022 & & 0.109 & 0.000 & 0.656 & \\
\hline
\end{tabular}

*SD; standard deviation; **Pearson correlation analysis, $\mathrm{p}<0.05$

Table 5: Distributions of PSQI subscales scores.

\begin{tabular}{|c|c|c|c|c|c|c|}
\hline \multirow[t]{2}{*}{ PSQI - Subscales } & \multicolumn{2}{|c|}{$\begin{array}{l}\text { Quality sleep } \\
\text { Good } \\
(n=84)\end{array}$} & \multicolumn{2}{|c|}{$\begin{array}{l}\text { Quality sleep } \\
\text { Poor } \\
\text { (n= 183) }\end{array}$} & \multicolumn{2}{|c|}{$\begin{array}{l}\text { Total } \\
\text { (Analysis) }\end{array}$} \\
\hline & n & $\%$ & $\mathbf{n}$ & $\%$ & $n$ & $\%$ \\
\hline Sleep quality & & & & & & $(U=11.75, p<0.001)$ \\
\hline Very good (0 pn) & 55 & 65.5 & 12 & 6.6 & 67 & 25.1 \\
\hline Very bad (3 pn) & 0 & 0.0 & 24 & 13.1 & 24 & 9.0 \\
\hline Sleep latency & & & & & & $(U=7.32, p<0.001)$ \\
\hline$<15 \min (0 \mathrm{pn})$ & 46 & 54.8 & 11 & 6.0 & 57 & 21.3 \\
\hline$>60 \min (3 \mathrm{pn})$ & 0 & 0.0 & 37 & 20.2 & 37 & 13.9 \\
\hline Sleep duration (Mean: $7.2 \pm 3.4$ ) & & & & & & $(U=8.51, p<0.001)$ \\
\hline$\geq 7$ hours (0 pn) & 72 & 85.7 & 81 & 44.3 & 153 & 57.3 \\
\hline$<5$ hours ( $3 \mathrm{pn})$ & 0 & 0.0 & 12 & 6.6 & 12 & 4.5 \\
\hline Sleep efficiency & & & & & & $(U=9.22, p<0.001)$ \\
\hline$>85 \%(0 \mathrm{pn})$ & 71 & 84.5 & 60 & 32.8 & 131 & 49.1 \\
\hline
\end{tabular}




\begin{tabular}{|l|l|l|l|l|l|l|}
\hline$<65 \%(3 \mathrm{pn})$ & 0 & 0.0 & 42 & 23.0 & 42 & 15.7 \\
\hline Sleep disturbance & & & & & & $(U=7.49, p<0.001)$ \\
\hline No (0 pn) & 0 & 0.0 & 0 & 0.0 & 0 & 0.0 \\
\hline$\geq 3$ times a week (3 pn) & 0 & 0.0 & 11 & 6.0 & 11 & 4.1 \\
\hline Sleep medication & & & & & & $(U=6.33, p<0.001)$ \\
\hline No (0 pn) & 84 & 100.0 & 182 & 99.5 & 266 & 99.6 \\
\hline$\geq 3$ times a week (3 pn) & 0 & 0.0 & 0 & 0.0 & 0 & 0.0 \\
\hline Daytime dysfunction & & & & & $(U=6.80, p<0.001)$ \\
\hline No (0 pn) & 70 & 83.3 & 27 & 14.8 & 97 & 36.3 \\
\hline$\geq 3$ times a week (3 pn) & 0 & 0.0 & 9 & 4.9 & 9 & 3.4 \\
\hline
\end{tabular}

*Mann Whitney $U$ test, $p<0.05$

Table 6: PSQI score distribution according to some characteristics of pregnant women.

\begin{tabular}{|c|c|c|c|c|c|c|c|c|c|}
\hline \multicolumn{10}{|c|}{ PSQI-Subscales $^{* *}$} \\
\hline \multicolumn{2}{|c|}{ Variables (analysis) } & 1 & 2 & 3 & 4 & 5 & 6 & 7 & $\begin{array}{l}\text { Global } \\
\text { Score }\end{array}$ \\
\hline \multirow{2}{*}{ Education } & $\mathrm{z}$ & 0.33 & 2.18 & 1.78 & 3.01 & 0.85 & 1.44 & 0.56 & 1.13 \\
\hline & $\mathrm{p}$ & 0.847 & 0.335 & 0.408 & 0.223 & 0.652 & 0.484 & 0.754 & 0.566 \\
\hline \multirow{2}{*}{ Age } & $\mathrm{z}$ & $6.57^{*}$ & 4.07 & $5.71^{*}$ & $8.17^{*}$ & $7.96^{*}$ & 2.60 & 4.86 & $6.10^{*}$ \\
\hline & $p$ & 0.034 & 0.252 & 0.074 & 0.042 & 0.046 & 0.456 & 0.182 & 0.011 \\
\hline \multirow{2}{*}{ Social support } & $U$ & $4.98^{*}$ & 0.85 & 1.09 & 1.81 & 1.90 & 0.47 & 0.44 & 1.21 \\
\hline & $p$ & 0.045 & 0.652 & 0.579 & 0.403 & 0.385 & 0.788 & 0.801 & 0.545 \\
\hline \multirow{2}{*}{ Income } & $\mathrm{z}$ & 1.28 & $9.23^{*}$ & $5.9^{*}$ & 1.75 & 1.05 & 0.75 & 0.84 & 5.05 \\
\hline & $p$ & 0.526 & 0.009 & 0.004 & 0.415 & 0.591 & 0.685 & 0.655 & 0.069 \\
\hline \multirow{2}{*}{ Chronic diseases } & $U$ & -1.80 & -0.91 & $-2.30^{*}$ & -1.72 & $-2.47^{*}$ & -0.61 & $-2.59^{*}$ & $-2.63^{*}$ \\
\hline & $p$ & 0.072 & 0.363 & 0.021 & 0.085 & 0.014 & 0.540 & 0.009 & 0.008 \\
\hline \multirow{2}{*}{ Obesity } & $z$ & $5.83^{*}$ & 0.80 & $6.16^{*}$ & $9.86^{*}$ & $5.87^{*}$ & 1.18 & 2.19 & $7.14^{*}$ \\
\hline & $p$ & 0.050 & 0.668 & 0.046 & 0.007 & 0.005 & 0.55 & 0.333 & 0.019 \\
\hline \multirow{2}{*}{ Gestational age } & $\mathrm{z}$ & $20.37^{\star}$ & $6.30^{*}$ & $9.53^{*}$ & $11.06^{*}$ & $14.76^{\star}$ & 2.98 & $6.84^{*}$ & $19.83^{*}$ \\
\hline & $p$ & 0.0001 & 0.041 & 0.009 & 0.004 & 0.0001 & 0.225 & 0.044 & 0.0001 \\
\hline \multirow{2}{*}{ Gravida } & $\mathrm{z}$ & 0.14 & $6.35^{*}$ & 3.22 & 1.67 & 1.57 & 2.81 & 2.08 & 4.06 \\
\hline & $\mathrm{p}$ & 0.147 & 0.042 & 0.201 & 0.433 & 0.45 & 0.245 & 0.352 & 0.135 \\
\hline \multirow{2}{*}{ Parity } & $\mathrm{z}$ & 3.24 & $7.72^{*}$ & 5.33 & 4.67 & 0.88 & 2.69 & 2.47 & 5.76 \\
\hline & $p$ & 0.125 & 0.021 & 0.063 & 0.126 & 0.65 & 0.261 & 0.291 & 0.052 \\
\hline \multirow{2}{*}{$\begin{array}{l}\text { Pregnancy } \\
\text { Relatedproblems }\end{array}$} & z & $-8.45^{\star}$ & $-7.00^{*}$ & $-5.64^{*}$ & $-5.76^{*}$ & $-7.20^{*}$ & -1.08 & $-6.45^{*}$ & $-9.31^{*}$ \\
\hline & $p$ & 0.0001 & 0.0001 & 0.0001 & 0.0001 & 0.0001 & 0.279 & 0.0001 & 0.0001 \\
\hline \multirow[t]{2}{*}{ Smoking } & $U$ & -1.72 & $-2.58^{*}$ & -1.36 & -0.63 & $-2.26^{*}$ & -0.45 & -1.87 & $-2.05^{\star}$ \\
\hline & $\mathrm{p}$ & 0.085 & 0.010 & 0.174 & 0.527 & 0.024 & 0.653 & 0.062 & 0.040 \\
\hline \multirow{2}{*}{$\begin{array}{l}\text { Drinking } \\
\text { Tea }\end{array}$} & $U$ & $7.86^{*}$ & 5.75 & 0.62 & 5.35 & 3.96 & 2.29 & 6.83 & 6.60 \\
\hline & $p$ & 0.050 & 0.190 & 0.892 & 0.152 & 0.266 & 0.513 & 0.077 & 0.086 \\
\hline \multirow{2}{*}{ Exercises } & $U$ & $-2.46^{*}$ & -0.26 & -1.50 & $-2.24^{*}$ & -1.23 & -0.64 & -1.22 & $-1.98^{*}$ \\
\hline & $p$ & 0.014 & 0.797 & 0.133 & 0.025 & 0.220 & 0.521 & 0.221 & 0.048 \\
\hline \multirow{2}{*}{ Antenatal care } & $U$ & $13.00^{*}$ & 4.655 & $9.422^{*}$ & 5.838 & 8.221 & 2.81 & 4.702 & $11.804^{*}$ \\
\hline & $p$ & 0.005 & 0.199 & 0.024 & 0.121 & 0.140 & 0.497 & 0.195 & 0.006 \\
\hline
\end{tabular}

*Mann Whitney $U$ and Kruskall Wallis analysis, $\mathrm{p}<0.05 ;{ }^{*}$ PSQI subscales; 1 = Sleep quality; 2 = Sleep latency; 3 = Sleep duration; 4 = Sleep efficiency; 5 = Sleep disturbance; 6 = Sleep medication; and 7 = Daytime function. 


\section{Discussion}

Pregnancy is a physiological challenge to virtually every organ system, in particular, the respiratory system. Thus, women are vulnerable to sleep disturbance during the perinatal period. A variety of sleep problems may worsen quality of life of the pregnant women [1-11]. In our study, it was determined that $68 \%$ of the women had insomnia. Recent studies also found that insomnia was common in the majority of pregnant women. These were as follows, Sedov, et al. (2018) 46\%, Kızılırmak, et al. (2012) 52.2\%, Coban and Yanıkkerem, et a. (2010) 54\%, Rezai, et al. (2013) 58\%, Dorheim, et al. 62\%, Kennelly, et al. (2011) 68\%, Lopez, et al. 70\%, Mindell, et al. (2015) 76\%, Smyka, et al. (2020) 77\%, Madevea, et al. (2014) 78\%, Wolyncyk-Gmaj, et al. (2017) $84.2 \%$, and Skoczlats, et al. (2014) 94.3\%, respectively.

Modifications in sleep pattern result from high circulating hormone levels and physical changes associated with pregnancy such as increased sympathetic activity, pulse rate, blood pressure and respiratory frequency with augmented alveolar oxygen gradient. All these changes contribute to the tiredness, fever, congestion, and respiration problems by the pregnant women $[26,29-34]$. We found that the most common reasons of insomnia were fatigue (being obliged to continue their traditional roles in the family), back-pain, growing their fetus, reflux, irritability, stress, fever, nausea, leg cramps and frequent urination. Similarly, previous studies showed that sleep problems were frequently associated with polyuria, fetal movements, uncomfortable position while sleeping, pain, breathing problems, and restless legs, respectively [8,21,22,26,35-37].

In the study, sleep quality has reduced significantly with increasing age $(p<0.05)$. According to the previous studies, advanced age was an important factor for insomnia $[3,4,8,25,26]$. Fatigue and other health problems that increase with age may cause sleep problems. In this study, it was found that uneducated women with poor income had more problems with sleep patterns $(p<0.05)$. Findings of this study are correlated with the literature $[3,5,26,37]$. However, Coban and Yanıkkerem, et al. (2010) did not detect a relationship between education level and quality of sleep in pregnant women. We found that sleep quality decreased significantly with the increase in the gestational age $(p<0.05)$. The related literature shows that insomnia is more common in the third trimester due to physical and psychosocial changes including anxiety, fetal growing, and respiratory distress [33-35].

In this study, obesity was an important modifier of sleep problems $(p<0.05)$. Obesity accompanied excessive soft tissue may narrow the pharyngeal airway and reduce lung volume. This situation may cause sleep problems along with apnea and snoring. Findings of this study are comparable with those in the literature [24,26,31,33-35].

Mental and physical discomfort caused by pregnancy related problems such as pain, interruption of sleep at night, waking up early in the morning, and being sleepy during the day may cause insomnia $[9,11,24,25]$. This study detected that the participants who had pregnancy-related complaints had the risk of developing insomnia more than those who did not $(p<0.05)$. However, our study revealed that most women did not go to the doctor for insomnia. Similarly, some authors reported that insomnia significantly linked with pregnancy-related problems $[15,16,18,21,25]$. Because of the complex and common co-morbid nature of insomnia, even if the problems related to pregnancy are minimized, the insomnia may not improve immediately. Several studies found that massage therapy was the non-pharmacological intervention most commonly preferred to pregnant women with low-back pain such as acupuncture, herbal, relaxation, exercise, yoga, and chiropractic, mindfulness, meditation, and physiotherapeutic counseling [17,36,37].

Significant relationship was detected between the state of smoking and insomnia $(p<0.05)$. Some authors identified that smoking pregnant women experienced sleep disturbances two times more frequently than those who did not $[26,30,31]$. In the present study, the score of sleep quality decreased significantly due to the stimulating effect of drinking tea $(p<0.05)$. The results of this study are consisted with other studies [33-35]. Physical activity is considered as a non-pharmacological approach to alleviating sleep problems [36]. In our study, sleep quality of those who exercise regularly during pregnancy was found to be better $(p<0.05)$. Similar result was obtained in previous studies $[37,38]$.

Although motherhood is a natural event, it brings along many difficulties. Thus, women are vulnerable to some problems about individual or family role functions during pregnancy $[3,7]$. In our study, women's quality of life of score was generally low (32 \pm 12.8$)$, particularly in the subscale of physical role function (Figure 1). Furthermore, there was significant inverse related between the quality of life score and insomnia $(p<0.05)$, so the $H_{1}$ hypothesis was confirmed. Similarly, recent researches indicated that the quality of life of pregnant women is related to their sleep quality $[1,4,6,10,25]$.

The validity of the findings is dependent on the individual's memory and accuracy in reporting sleep problems. The sample in this study reflects only one area of Turkey and the findings should be limited to this population. However, our findings highlight the importance of considering the sociodemographic, medical, pregnancy-related characteristics and habits for each woman with pregnant to ensure correct approach for alleviating sleep problems. Also, the data of this cross-sectional study were based on clinically prognostic scale of the women by face-to-face interview instead of retrospective patient chart based reports.

\section{Conclusion}

Sleep problems was common in the majority of pregnant women. Also, quality of life decreased with increasing insomnia. In line with these findings, women should be thoroughly evaluated for insomnia symptoms including risk factors as part of usual antenatal care and treated timely with effective strategies such as improving sleep hygiene, behavioral therapies, and pharmacotherapy by healthcare professionals. Future research with larger group should examine various factors underlying poor sleep quality during pregnancy to improve women's quality of life. 


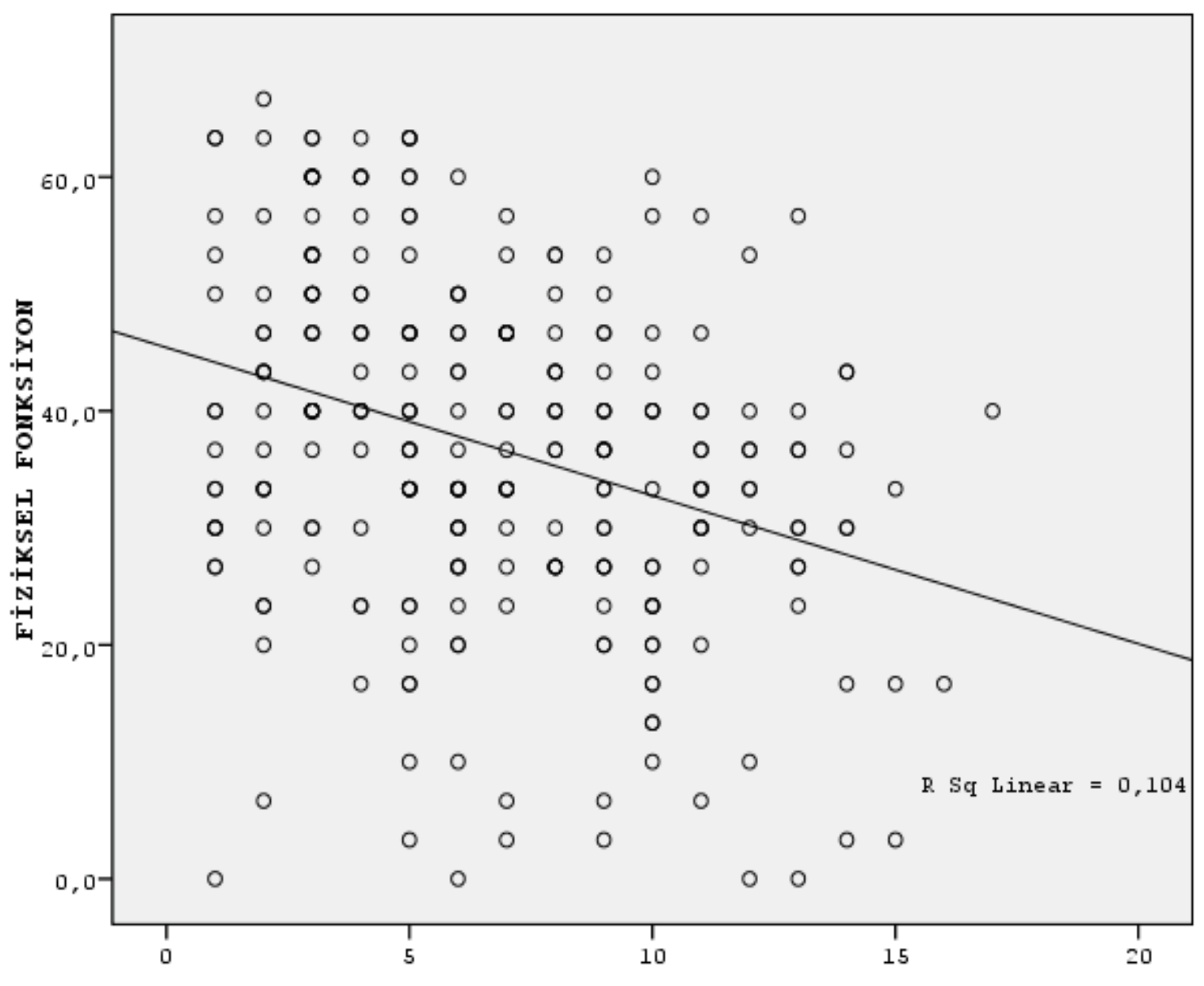

Global PSQI ( Pitssburg Uyku Kalitesi İndeksi)

Figure 1: Physical function distribution graph with PSQI.

\section{Acknowledgments}

We would like to thank the pregnant women who supported us during the data collection.

\section{Conflict of Interest}

The authors have no conflicts of interest.

\section{Funding}

The author(s) received no financial support for the research, authorship, and/or publication of this article.

\section{References}

1. Mogos MF, August EM, Salinas-Miranda AA, et al. (2013) Systematic review of quality of life measures in pregnant and postpartum mothers. Appl Res Qual Life 8: 219-250.

2. Strine TW, Chapman DP (2005) Associations of frequent sleep insufficiency with health-related quality of life and health behaviors. Sleep Med 6: 23-27.

3. Rezai E, Moghadam ZB, Saraylu K (2013) Quality of life in pregnant women with sleep disorder. J Family Reprod Health 7: 8793.
4. Dall'alba V, Callegari-Jacques SM, Krahe C, et al. (2015) Health-related quality of life of pregnant women with heartburn and regurgitation. Arq Gastroenterol 52: 100-104.

5. Bazalakova M (2007) Sleep Disorders in Pregnancy. Semin Neurol 37: 661-668.

6. Dixon CL (2014) Sleep deprivation and pregnancy related risks. Int J Childbirth Educ 29: 62-65.

7. Madaeva IM, Kolesnikova LI, Protopopova NV et al. (2014) Features of the sleep pattern during pregnancy. Vest Ross Akad Med Nauk (1-2): 93-97.

8. Mindell JA, Cook RA, Nikolovski J (2015) Sleep patterns and sleep disturbances across pregnancy. Sleep Med 16: 483-488.

9. Okun ML, Buysse DJ, Hall MH (2015) Indentifying insomnia in early pregnancy: Validation of the insomnia symptoms questionnaire (ISQ) in pregnant women. J Clin Sleep Med 11: 645-654.

10. Plancoulaine S, Flori S, Bat-Pitault F, et al. (2017) Sleep trajectories among pregnant women and the impact on outcomes: a population-based cohort study. Matern Child Health J 21: 11391146.

11. Hashmi AM, Bhatia SK, Bhatia SK, et al. (2016) Insomnia during pregnancy: Diagnosis and rational interventions. Pak J Med Sci 32: 1030-1037. 
12. Reichner CA (2015) Insomnia and sleep deficiency in pregnancy. Obstet Med 8: 168-171.

13. Reid KJ, Facco FL, Grobman WA, et al. (2017) Sleep during pregnancy: The nuMoM2b pregnancy and sleep duration and continuity study. Sleep 40: zsx045.

14. Sedov ID, Cameron EE, Madigan S, et al. (2018) Sleep quality during pregnancy: A meta-analysis. Sleep Med Rev 38: 168-176.

15. Román-Gálvez RM, Amezcua-Prieto C, Salcedo-Bellido I, et al. (2018) Factors associated with insomnia in pregnancy: A prospective cohort study. Eur J Obstet Gynecol Reprod Bio 221: 70 75.

16. Sharma SK, Nehra A, Sinha S, et al. (2016) Sleep disorders in pregnancy and their association with pregnancy outcomes: A prospective observational study. Sleep Breath 20: 87-93.

17. Truong KK, Guilleminault C (2018) Sleep disordered breathing in pregnant women: Maternal and fetal risk, treatment considerations, and future perspectives. Expt Rev Resp Med 12: 177-189.

18. Umeno S, Kato C, Nagaura Y, et al. (2020) Characteristics of sleep/wake problems and delivery outcomes among pregnant Japanese women without gestational complications. BMC Pregnancy Childbirth 20: 179-184.

19. Wang L\& Jin F (2020) Association between maternal sleep duration and quality, and the risk of preterm birth: A systematic review and meta-analysis of observational studies. BMC Pregnancy Childbirth 20: 125-30.

20. Warland J, Dorrian J, Morrison JL, et al. (2018) Maternal sleep during pregnancy and poor fetal outcomes: A scoping review of the literature with meta-analysis. Sleep Med Rev 41: 197-219.

21. Smyka M, Kaczynska KK, Wojcicka NS, et al. (2020) Sleep problems in pregnancy-a cross-sectional study in over 7000 pregnant women in Poland. Int J Environ Res Public Health 17: 5306.

22. Skoczylas M, Łęgowik A, Krawczyk P, et al. (2014) Sleeping disorders among pregnant woman. Gin Pol Med Project 4: 34-40.

23. Silvestri R, Arico I (2019) Sleep disorders in pregnancy. Sleep Sci 12: 232-239.

24. Wołyńczyk-Gmaj D, Różańska-Walędziak A, Ziemka, et al. (2017) Insomnia in pregnancy is associated with depressive symptoms and eating at night. Clin Sleep Med 13: 1171-1176.
25. Coban A, Yanıkkerem UE (2010) Sleep quality and fatigue in pregnant women. Ege Journal of Medicine 49: 87-94.

26. Kızılırmak A, Timur S, Kartal B (2012) Insomnia in pregnancy and factors related to insomnia. The Scie World J 2: 1-8.

27. Ozdamar K (2003) Modern scientific research methods. Kaan Bookstore, Eskisehir, 117.

28. Buysse DJ, Reynolds CF, Monk TH, et al. (1989) The pittsburgh sleep quality index (PSQI): A new instrument for psychiatric research and practice. Psychiatry Research 28: 193-213.

29. RAND (2009) Medical outcomes study: 36-item short form survey scoring instructions.

30. Da Costa D, Dritsa M, Verreault N, et al. (2010) Sleep problems and depressed mood negatively impact health-related quality of life during pregnancy. Arch Womens Ment Health 13: 249-257.

31. Facco FL, Grobman WA, Reid KJ, et al. (2017) Objectively measured short sleep duration and later sleep midpoint in pregnancy are associated with a higher risk of gestational diabetes. Am J Obstet Gynecol 217: 447.E1-447.E13.

32. Dorheim SK, Bjorvatn B, Eberhard-Gran M (2012) Insomnia and depressive symptoms in late pregnancy: $A$ population-based study. Behav Sleep Med 10: 152-166.

33. Fernandez-Alfonson AM, Trabalon-Pastor M, Peter Chedraui, et al. (2012) Factors related to insomnia and sleepiness in the late third trimester of pregnancy. Arch Gynecol Obstet 286: 55-61.

34. Kennelly MM, Fallon A, Farah N, et al. (2011) Effects of body mass index on sleep patterns during pregnancy. J Obstet Gynaecol 31: 125-127.

35. Palagini L, Gemignani A, Banti S, et al. (2014) Chronic sleep loss during pregnancy as a determinant of stress: Impact on pregnancy outcome. Sleep Med 15: 853-859.

36. Hollenbach D, Broker R, Herlehy SH, et al. (2013) Non-pharmacological interventions for sleep quality and insomnia during pregnancy: A systematic review. J Can Chiropr Assoc 57: 21-31.

37. Hutchison BL, Stone PR, McCowan LM, et al. (2012) A postal survey of maternal sleep in late pregnancy. BMC Pregnancy and Childbirth 12: 144-148.

38. Tella BA, Sokunbi OG, Akinlami OF, et al. (2011) Effects of aerobic exercises on the level of insomnia and fatigue in pregnant women. Int J Gyn Obst 15: 75-80.

DOI: $10.36959 / 545 / 397$

Copyright: (c) 2021 Celikgoz D, et al. This is an open-access article distributed under the terms of the Creative Commons Attribution License, which permits unrestricted use, distribution, and reproduction in any medium, provided the original author and source are credited. 